\title{
Safety And Efficacy of Transjugular Intrahepatic Portosystemic Shunt Creation For Hepatic Hydrothorax: A Retrospective Review
}

\author{
Carson Powell ${ }^{1}$, Paul Haste ${ }^{2}$ \\ Indiana University School of Medicine ${ }^{1}$, Department of Radiology \& Imaging Sciences²
}

Background and Objective: Hepatic hydrothorax is a type of pleural effusion that occurs in 5$10 \%$ of patients with poor liver function. This causes patients to experience shortness of breath and chest discomfort with the potential for respiratory or cardiovascular collapse. When medical management fails, thoracentesis to directly drain fluid is a second-line therapy but frequent treatment is not recommended. For patients requiring serial thoracentesis, a transjugular intrahepatic portosystemic shunt (TIPS) may relieve hepatic hydrothorax by creating a connection between the portal vein and hepatic vein, thus reducing portal hypertension and decreasing fluid accumulation. Our objective was to assess the safety and efficacy of TIPS for hepatic hydrothorax.

Methods: An IRB approved, HIPAA compliant institutional database of 320 patients who underwent TIPS creation between 2011-2016 was retrospectively reviewed for patients whose primary indication for TIPS creation was hepatic hydrothorax. This yielded a total of 24 individual patients. Records were reviewed and evaluated for overall survival (OS), response (as measured by persistent need for thoracentesis), and frequency of new and/or worsening hepatic encephalopathy (HE).

Results: Survival was $92 \%, 62 \%$ and $38 \%$ for 30 days, 1 year, and 3 year survival, respectively. Response to TIPS was graded as complete (52\%), partial (19\%), or unresponsive $(29 \%)$ for an overall response rate of $71 \%$. $29 \%$ of patients required TIPS revision. $24 \%$ of patients experienced new or worsened hepatic encephalopathy that was successfully managed with medication in all but 1 case. Analysis of relationships between response grades, 1 year survival, and age older vs younger than 60 years did not have statistically significant relationships between MELD scores, Child-Pugh scores, post-TIPS pressure gradients, and serological data.

Conclusion: This study reaffirms the safety and efficacy of TIPS for hepatic hydrothorax but further work must be done to identify ideal candidates and prognostic factors for this procedure. 